\title{
BMJ Open Asymptomatic bacteriuria in pregnancy: systematic reviews of screening and treatment effectiveness and patient preferences
}

\author{
Aireen Wingert, ${ }^{\oplus 1}$ Jennifer Pillay, ${ }^{1}$ Meghan Sebastianski, ${ }^{2}$ Michelle Gates, ${ }^{\oplus 1}$ \\ Robin Featherstone, ${ }^{1,2}$ Kassi Shave, ${ }^{1}$ Ben Vandermeer, ${ }^{1}$ Lisa Hartling ${ }^{1,2}$
}

To cite: Wingert A, Pillay J, Sebastianski M, et al. Asymptomatic bacteriuria in pregnancy: systematic reviews of screening and treatment effectiveness and patient preferences. BMJ Open 2019;9:e021347. doi:10.1136/ bmjopen-2017-021347

- Prepublication history and additional material for this paper are available online. To view these files, please visit the journal online (http://dx.doi org/10.1136/bmjopen-2017021347).

Received 26 December 2017 Revised 16 August 2018 Accepted 5 February 2019

Check for updates

(c) Author(s) (or their employer(s)) 2019. Re-use permitted under CC BY-NC. No commercial re-use. See rights and permissions. Published by BMJ.

${ }^{1}$ Pediatrics, University of Alberta, Alberta Research Centre for Health Evidence (ARCHE), Edmonton Clinic Health Academy, Edmonton, Alberta, Canada

${ }^{2}$ Alberta Strategy for PatientOriented Research (SPOR) SUPPORT Unit Knowledge Translation Platform, University of Alberta, Edmonton, Alberta, Canada

Correspondence to

Lisa Hartling;

hartling@ualberta.ca

\section{ABSTRACT}

Objective To systematically review screening and treatment effectiveness, and patient preferences, to inform recommendations by the Canadian Task Force on Preventive Health Care on screening for asymptomatic bacteriuria in pregnancy.

Design We searched multiple databases (inception to September 2017) and grey literature sources for studies on screening effectiveness and patient preferences. For treatment with antibiotics, we searched three databases for systematic reviews and obtained search results of the Cochrane Pregnancy and Childbirth Group's Trials Register to update a Cochrane review. Study selection, risk of bias assessment and evaluation of the quality for each outcome using Grading of Recommendations Assessment, Development and Evaluation was completed independently by two reviewers with consensus. Meta-analysis was conducted when appropriate as were analyses based on planned subgroup variables.

Outcomes For screening and treatment effectiveness: maternal and perinatal mortality, maternal and neonatal sepsis, pyelonephritis, spontaneous abortion, preterm delivery, low birth weight and serious adverse events. Valuation of outcomes for patient preferences.

Results Four studies compared outcomes before and after the introduction of a screening programme or between different screening programmes. All evidence on screening effectiveness was considered very low quality. Women have conflicting opinions about antibiotic use during pregnancy. Fifteen trials compared antibiotic treatment with no treatment or placebo in women with confirmed bacteriuria. Low-quality evidence found that treatment lowered rates of pyelonephritis (12 trials, relative risk [RR] $0.24 ; 95 \% \mathrm{Cl} 0.13$ to 0.42 ; absolute risk reduction [ARR] 17.6\%; number needed to treat [NNT] 6, 95\% Cl 5 to 7) and low birth weight (seven trials, RR $0.63 ; 95 \% \mathrm{Cl} 0.45$ to 0.90 ; ARR $4.4 \%$; NNT 23, $95 \% \mathrm{Cl} 15$ to 85 ).

Conclusions Antibiotic treatment for women having significant bacteriuria likely reduces the incidence of pyelonephritis and low birth weight, but we are uncertain about the magnitude of the effect and about the extent to which we can apply these results to asymptomatic populations and screening programmes. PROSPERO registration number CRD42016045263.
Strengths and limitations of this study

- Comprehensive search, risk of bias and quality assessments were conducted for all studies.

- Methodological limitations were common across many studies.

- Applicability of results to routine, prenatal care for women is uncertain due to scant and inconsistent reporting of population and screening characteristics among included studies.

- The quality of the body of evidence was low to very low for reported outcomes.

- No direct evidence was available on how women weigh benefits and harms of screening.

\section{BACKGROUND}

Asymptomatic bacteriuria (ASB) signifies a significant quantitative count of bacteria in the urine without symptoms of a lower (acute cystitis) or upper urinary tract/kidney (acute pyelonephritis) infection (UTI). ${ }^{1}{ }^{2}$ Prevalence of ASB in premenopausal, ambulatory women is $2 \%-10 \%,{ }^{1}$ but due to anatomical and physiological changes (eg, displaced bladder) to the urinary tract in pregnancy, there are theoretical reasons to suspect higher rates of ASB during pregnancy and consequently a greater chance of progression to symptomatic UTI and other pregnancy complications (eg, pyelonephritis, preterm delivery). ${ }^{13}$ Numerous risk factors for ASB in pregnancy have been identified (eg, low socioeconomic status, higher parity, a history of recurrent UTI, diabetes and anatomical abnormalities of the urinary $\operatorname{tract}^{124}$ ).

Controversy exists over the mechanism linking ASB, pyelonephritis and adverse perinatal outcomes (ie, whether ASB affects pregnancy and neonatal outcomes solely through pyelonephritis or also other mechanisms such as prostaglandin activation), ${ }^{24}$ and therefore also about whether treatment of ASB with 
antibiotics will reduce the risk of such adverse outcomes. Additionally, some sources have outlined concerns with incidence and reporting on adverse effects of antibiotic treatment for ASB, UTIs or antibiotic use in general during pregnancy. ${ }^{245}$

Reports of reduced incidence of pyelonephritis in pregnant women after introduction of routine screening (eg, $0.3 \%-0.57 \%$ vs $1 \%-2 \%{ }^{6}$ ) suggest that these programmes have been beneficial. Practices of urine testing may be used to detect conditions in pregnancy other than ASB. There appears to be diversity in screening for ASB with variations in urine testing methods, timing, and collection, as well as treatment protocols (duration, test for cure, threshold of bacteria for treatment).

The findings from this review were used by the Canadian Task Force on Preventive Health Care (CTFPHC) supplemented by consultations with patients on outcome valuation and by information from stakeholders and other sources on issues of feasibility, acceptability, costs/ resources and equity-to inform recommendations on screening for ASB to support primary healthcare providers in delivering preventive care (available at http://www.cmaj.ca/content/190/27/E823). A staged approach to the research questions was used, beginning with an examination of direct evidence on the effectiveness of screening programmes and of women's outcome valuation:

1. What are the benefits and harms of screening compared with no screening, or different screening methods or algorithms, for ASB in pregnancy?

Screening is a programme, not only a test. Screening, therefore, includes a series of events initiated by systematically offering a test to diagnose ASB in all asymptomatic pregnant women, with subsequent decisions about and adherence to treatment protocols and any other follow-up activities.

2. How do women weigh the benefits and harms of screening and treatment of ASB in pregnancy, and how does this outcome valuation inform their decisions to undergo screening?

If there was insufficient quality of evidence from screening effectiveness and women's outcome valuation for the CTFPHC to make a recommendation, an examination of treatment effectiveness (one key component of a screening programme) is planned:

3. What are the benefits and harms of antibiotic treatment compared with placebo or no treatment for ASB in pregnancy?

\section{METHODS}

This series of systematic reviews (SRs) follow methods of the CTFPHC; the protocol was registered with PROSPERO (CRD42016045623) and is available in online supplementary 1 . As per our staged approach, all steps were conducted for the questions on screening effectiveness and patient preferences before it was determined (due to very low quality evidence on screening based on the Grading of Recommendations Assessment, Development and Evaluation [GRADE]) to conduct the review on treatment effectiveness.

\section{Patient and public involvement}

The CTFPHC incorporates patient preferences at three critical points in the guideline development process: (1) when outcomes are selected for inclusion in the SR protocol; (2) when the final guideline recommendations and knowledge translation (KT) tool are being developed and (3) when patient KT tools are tested for usability. Relevant to this review, online surveys and telephone focus groups were conducted with women $(n=17 ; 10$ of whom were pregnant) by an independent group with expertise in KT from St. Michael's Hospital (Toronto, Ontario) to obtain ratings of all potential outcomes and input on how consideration of these outcomes would influence their decision to be screened. All outcomes rated as critical (7-9 out of 9) or important (4-6 out of 9) for these women's decision-making were considered for inclusion in the SRs on screening and treatment. Other components of the research design and the conduct of the reviews did not involve patients or the public.

\section{Search strategy}

Comprehensive searches were developed and conducted by a research librarian for each question (details in online supplementary 2). For screening effectiveness, the following databases were searched in June 2016 and then updated in September 2017: Ovid MEDLINE (1946-); Ovid Embase (1974-); Wiley Cochrane Library (inception-); CINAHL via EBSCOhost (1937-) and PubMed via NCBI Entrez (1946-). For women's outcome valuation, we also searched Ovid PsycINFO (1806-). For all searches, limits were applied for language (English and French) but not publication date. For treatment effectiveness, SRs were searched in October 2016 in PubMed, the Cochrane Database of Systematic Reviews and the Database of Abstracts of Reviews of Effects (both via Cochrane Library). Authors of the SR on treatment ${ }^{4}$ provided results of their recent search update (Cochrane Pregnancy and Childbirth Group's Trials Register) in October 2017. Additional studies were identified through contact with experts and recommended grey literature sources. ${ }^{7}$

\section{Study selection and eligibility criteria}

Two reviewers independently screened titles and abstracts, followed by full-text review, using a standard eligibility criteria form and DistillerSR software (Evidence Partners, Ottawa, Canada). ${ }^{8}$ The flow of screening and decisions were recorded in a Preferred Reporting Items for Systematic Reviews and Meta-Analyses flow chart.

The criteria for inclusion (populations, interventions, comparators, outcomes, timing and setting [PICOTS]) for screening and treatment effectiveness, and women's outcome valuation are summarised in online supplementary 3 . 
For treatment effectiveness, existing SRs were eligible based on (1) searching more than one database, (2) reporting selection criteria and (3) using PICOTS criteria that closely match the current review. The included studies were assessed for eligibility to meet our inclusion criteria, incorporating existing data and extracting additional data as necessary, conducting quality assessments and performing new meta-analyses and GRADE quality assessments.

\section{Data extraction and risk of bias assessment}

One reviewer independently extracted data and another verified data from each included study on study details and relevant PICOTS, including information for patient and intervention subgroups. Authors of included studies were contacted for clarification of study details and outcome data with follow-up as necessary. Intention-totreat results were recorded whenever possible. For all outcomes, including harms, counts or proportions and sample size by study arm, were recorded.

Two reviewers independently assessed the risk of bias (ROB) of each included study with disagreements resolved via consensus or third reviewer consultation. For observational studies on screening effectiveness, the Newcastle-Ottawa Quality Assessment Scale was used; a separate assessment for reporting bias was included due to suspected selective outcome reporting. For cross-sectional studies on women's outcome valuation, the tool developed by the Centre for Evidence-based Management ${ }^{9}$ was used. For treatment effectiveness, all controlled trials were appraised using the Cochrane ROB tool. ${ }^{10}$

\section{Data synthesis and analysis}

Relative risks (RRs) were reported using the DerSimonian and Laird random-effects model with Mantel-Haenszel method and corresponding 95\% CI. Sensitivity (for ROB and study design) and subgroup (for predefined population and screening characteristic variables) analyses were conducted when possible and appropriate. We report values for statistical heterogeneity $\left(\mathrm{I}^{2}\right)$ but did not rely on this for decisions about meta-analysis or subgroup analysis. A minimum of two of the following criteria determined credibility of subgroup investigations: (1) visual inspection of forest plot showing a meaningful difference between effect estimates (eg, clinical decision-making on the intervention would differ for each subgroup), (2) a reduction in the heterogeneity $\left(\mathrm{I}^{2}\right)$ for each subgroup from the original meta-analysis and (3) a statistically significant $\chi^{2}$ test for subgroup effects.

Analyses were performed using Review Manager V.5.3. For outcomes that demonstrated significant effects, absolute risk reduction (ARR) and number needed to screen (NNS) or number needed to treat (NNT), were calculated. The values for NNS or NNT were calculated using absolute numbers from the GRADE tables estimated using the control group event rate and RR with the $95 \%$ CI obtained from the meta-analysis. ${ }^{11}$

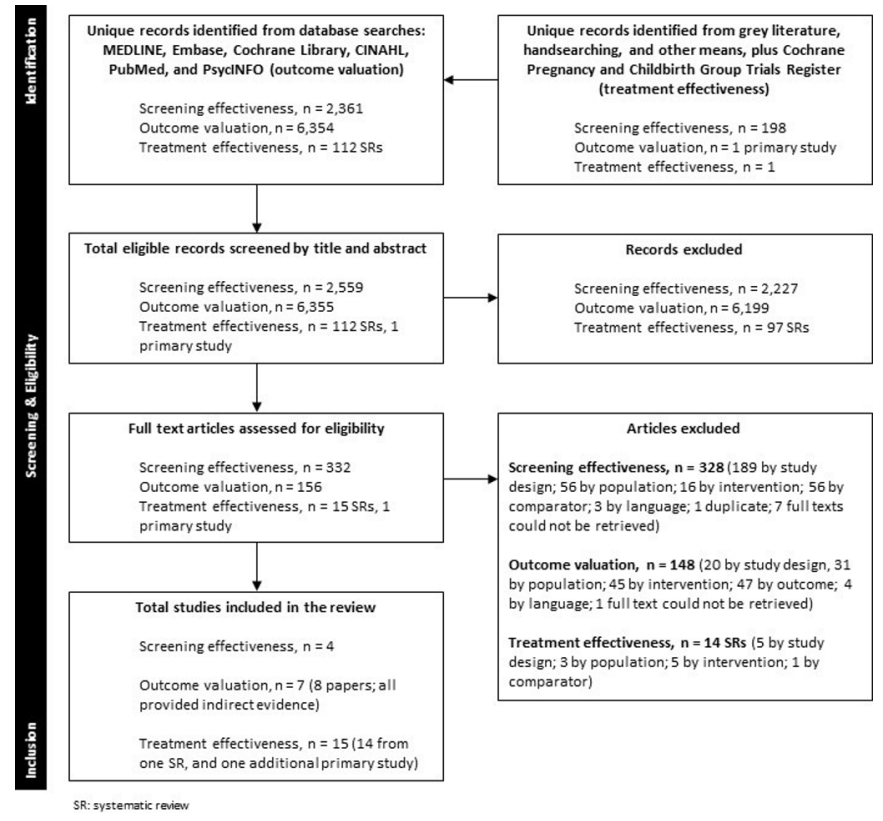

Figure 1 PRISMA flow diagram of study selection. PRISMA, Preferred Reporting Items for Systematic Reviews and MetaAnalyses.

Small-study bias (for meta-analyses with eight or more studies) was assessed using the funnel plot and Egger's test. $^{12}$

\section{Quality assessment}

Two reviewers independently assessed the quality of the body of evidence for each outcome using GRADE methodology $^{1314}$ with disagreements resolved through discussion or consultation with a third reviewer. For evidence on benefits and harms of screening and treatment, quality was assigned initially as high for evidence from randomised controlled trials (RCTs) and low for evidence from observational studies. Thereafter, quality was potentially downgraded based on five core domains: study limitations/ROB, inconsistency, indirectness, imprecision and publication/reporting bias. We did not consider upgrading because of serious concerns with the main domains. ${ }^{15}$ Assessments were entered into the GRADEpro software $^{16}$ and summarised in GRADE summary of findings (SOF) and evidence profiles (EP) tables. ${ }^{17}$

\section{RESULTS}

\section{Study selection and characteristics}

Study flow and selection is in figure 1. A total of 25 unique studies were included in the review. Characteristics of included studies for screening and treatment effectiveness are in table 1; detailed study information is in online supplementary 4 .

Screening effectiveness: four studies (7611 women) ${ }^{18-21}$ were included. One study ${ }^{18}$ was published in French. All were non-concurrent cohort studies, comparing outcomes before and after introduction of a screening programme. 


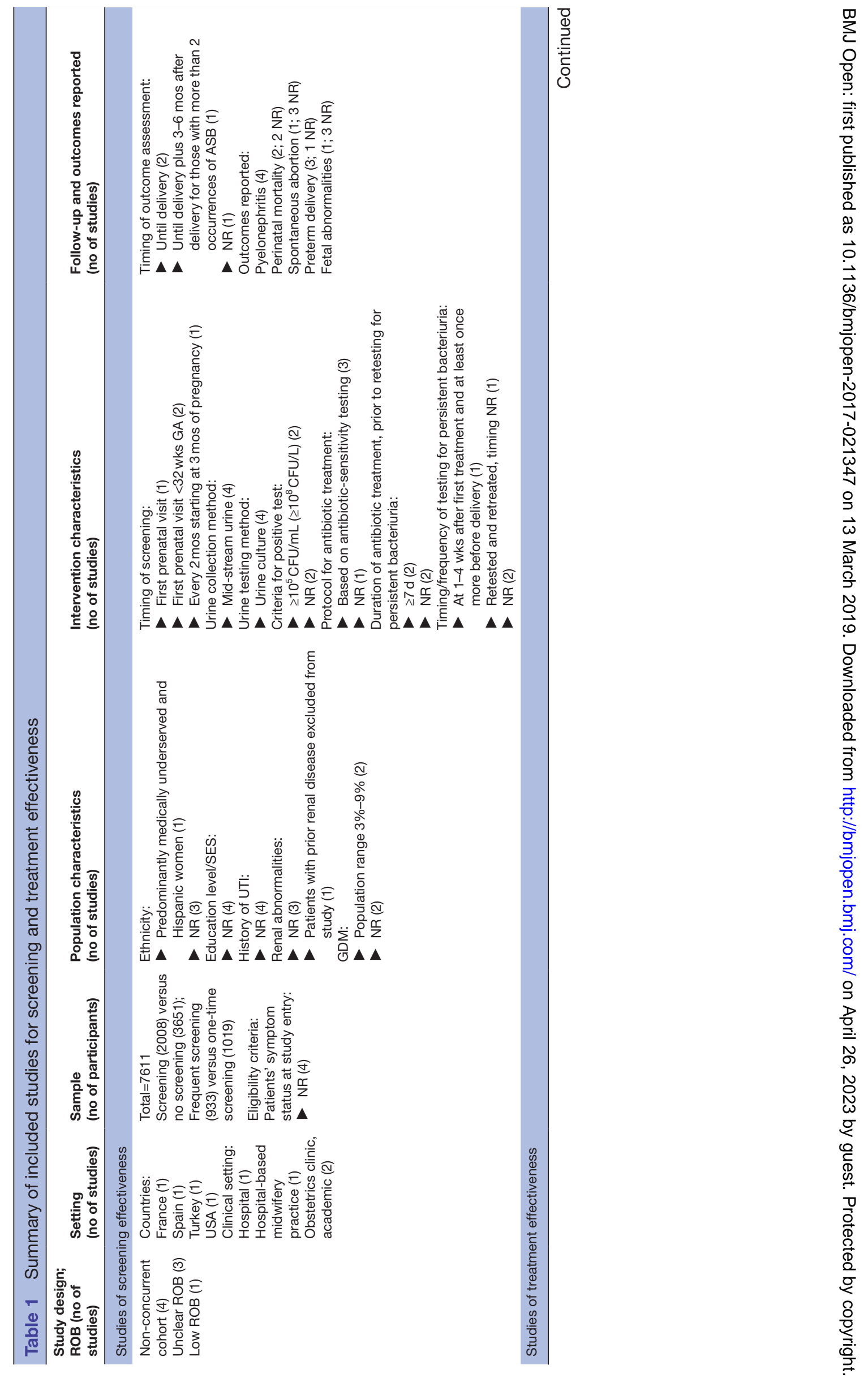




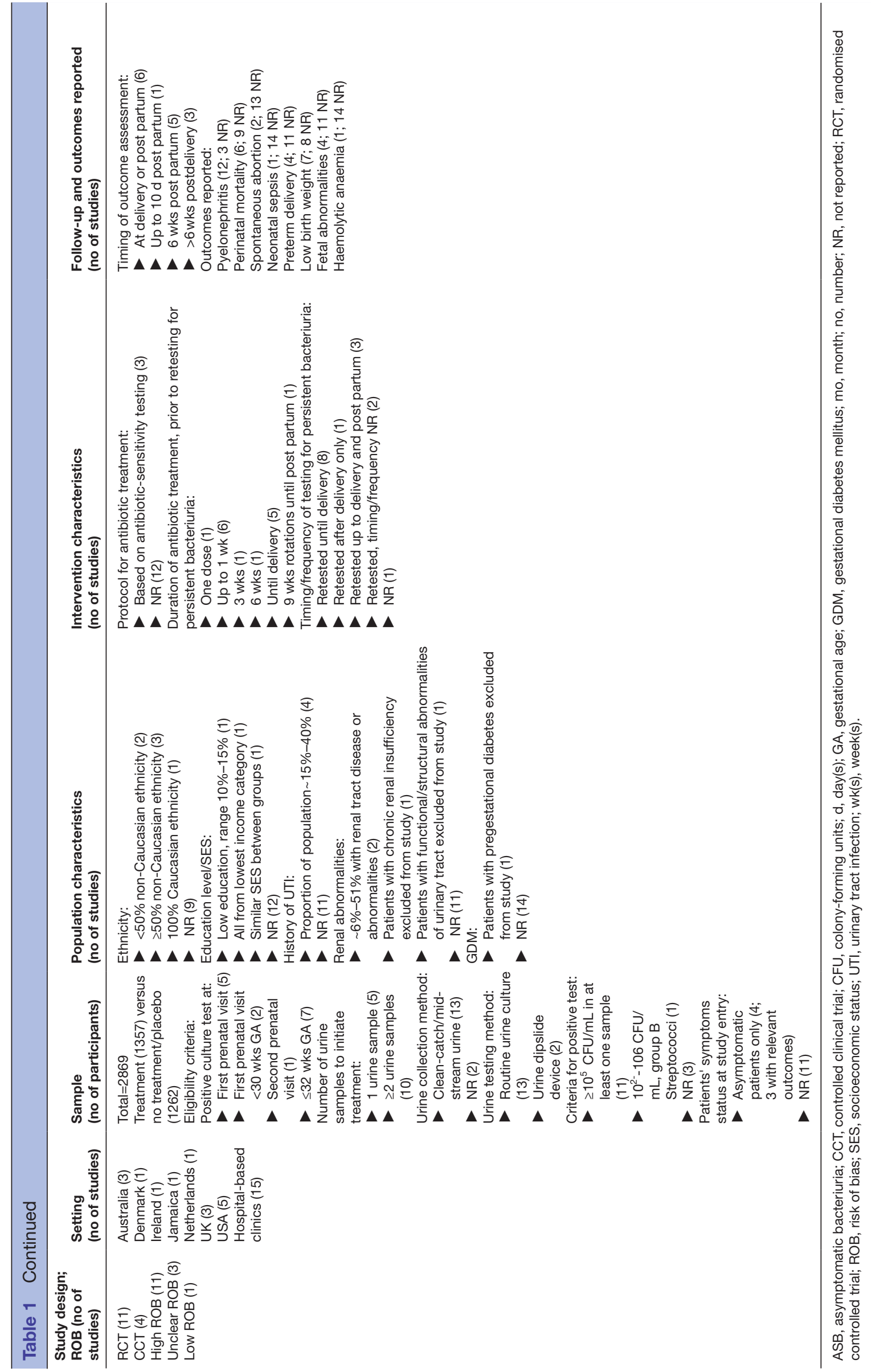


Women's outcome valuation: no studies were identified that examined how women weigh the benefits and harms of screening and/or treatment of ASB in pregnancy or how their valuation of benefits and harms inform their decisions to undergo screening and treatment. Six surveys and one cross-sectional study were included: three studies provide information on drug utilisation opinions, ${ }^{22-24}$ while four studies provide information on perceptions of teratogenic risk. ${ }^{25-29}$ One study was a multicentre screening cohort of pregnant women with an embedded RCT of antibiotic treatment for women with significant bacteriuria; cross-sectional findings from the women eligible for treatment are used for information on treatment preference. ${ }^{23}$

Treatment effectiveness: one $\mathrm{SR}^{4}$ met our inclusion criteria. Contact with the information specialist of the Cochrane Pregnancy and Childbirth Group's Trials Register confirmed one study (Kazemier et $a l^{23}$ ) identified from their ongoing search updates (to October 2017) relevant for treatment effectiveness. Fifteen primary studies ${ }^{23} 30-43$ (2869 women), mostly published in the 1960s, examined treatment effectiveness for bacteriuria. One study ${ }^{31}$ included in the Cochrane review ${ }^{4}$ only reported on persistent bacteriuria and therefore was excluded from analysis and the overall body of evidence relevant to our outcomes of interest.

\section{ROB and quality assessments}

Overall ROB assessments for screening and treatment effectiveness are in table 1 , and reported with quality assessments below; detailed ROB assessments are in online supplementary 5 .

For women's outcome valuation, while all seven studies addressed a focused research question and used a sample representative of this study question, their reported sampling methods could potentially introduce bias and only one of the studies ${ }^{25}$ fully accounted for confounding factors through statistical analysis. None of the papers reported that their sample size was based on prestudy considerations while only two papers ${ }^{24}{ }^{29}$ used survey questions that were considered valid and reliable.

Quality of evidence assessments for screening and treatment effectiveness are in table 2; detailed GRADE SOF and EP tables and forest plots are in online supplementary 6 .

\section{Screening effectiveness}

Three studies ${ }^{18} 1921$ of unclear ROB (5659 women) found a statistically significant difference for screening compared with no screening on the outcome of pyelonephritis (RR 0.28 ; $95 \%$ CI 0.15 to $0.54 ; \mathrm{I}^{2}=0 \%$; ARR $1.3 \%$; NNS 77, 95\% CI 65 to 121; very low quality). One study ${ }^{20}$ (1952 women) with low ROB comparing screening at all prenatal visits with a screening at first prenatal visit only, found no significant difference for pyelonephritis (RR 1.09 ; $95 \%$ CI 0.27 to 4.35 ; very low quality).

Two studies ${ }^{12}$ (724 women) with unclear ROB and suspected reporting bias ${ }^{18}$ found no significant difference
(RR $1.21,95 \%$ CI 0.01 to $102.93, \mathrm{I}^{2}=84 \%$; very low quality) in perinatal mortality.

One study of 370 women ${ }^{18}$ with unclear ROB but suspected reporting bias found no significant difference (RR $0.96,95 \%$ CI 0.41 to 2.27 ; very low quality) in spontaneous abortion at $\leq 28$ weeks of gestation.

Two studies ${ }^{18} 21$ (722 women) with unclear ROB but suspected reporting bias ${ }^{18}$ compared screening with no screening and found no significant difference (RR 8.70, $95 \%$ CI 0.32 to $240.07 ; \mathrm{I}^{2}=80 \%$; very low quality) in preterm delivery. The study ${ }^{20}$ comparing different screening algorithms found a significant difference for preterm delivery (RR 1.57 ; $95 \%$ CI 1.11 to 2.23 ; very low quality) with more preterm deliveries among the group that was screened at all prenatal visits. The study authors did not present a possible hypothesis to explain this result.

One study ${ }^{21}$ (372 women) with unclear ROB found no significant difference (RR 1.50, 95\% CI 0.25 to 8.87; very low quality) in fetal abnormalities.

No study reported on maternal mortality, maternal sepsis, neonatal sepsis or low birth weight.

Subgroup analyses were not performed due to an insufficient number of studies per category comprising a priori subgroups.

\section{Women's outcome valuation}

Studies demonstrated varied opinions on antibiotic use during pregnancy, with nearly half of participants from two studies (47\%-48\%) expressing that antibiotics should not be used during pregnancy. ${ }^{22}{ }^{24}$ Cross-sectional analysis of patients recruited for an RCT of treatment for ASB found similar results, with $61 \%$ of 255 women with ASB not wanting to be treated for an asymptomatic condition. ${ }^{23}$ Some evidence suggested that women thought penicillin posed a teratogenic risk ${ }^{25} 27$ and that antibiotics were unsafe during pregnancy, particularly for the fetus. ${ }^{26}{ }^{28}$ How these attitudes may inform the women's decisions on whether to screen for ASB was not reported, nor were details on accuracy or understanding of information regarding potential risks and benefits.

\section{Treatment effectiveness}

Twelve studies 2330 32-40 42 (2017 women) examined the effects of antibiotic treatment and found a significant difference in development of pyelonephritis (RR 0.24; $95 \%$ CI 0.13 to $0.41 ; \mathrm{I}^{2}=60 \%$; ARR $17.6 \%$; NNT $6,95 \%$ CI 5 to 7 ; low quality) (figure 2). Three trials explicitly included women without symptoms at baseline (other trials may have included some symptomatic women); sensitivity analysis did not affect the results (three trials, ${ }^{23} 3942 \mathrm{RR}$ $0.22 ; 95 \%$ CI 0.10 to $0.49 ; \mathrm{I}^{2}=0 \%$ ). Sensitivity analysis for ROB (removing studies with overall high risk) and study design (removing CCTs) did not change the results.

Subgroup analysis for the number of urine samplesstudies using one or more additional cultures to confirm ASB compared with just one culture-appeared to explain the heterogeneity among all studies combined $\left(\mathrm{I}^{2}=60 \%\right.$ ) for pyelonephritis (RR $0.19,95 \%$ CI 0.11 to 0.31 ; 
Table 2 Summary of findings for effects of screening programmes and of treatment on maternal and neonatal benefits and harms

\section{Screening versus no screening}

\begin{tabular}{|c|c|c|c|c|c|}
\hline \multirow[b]{2}{*}{ Outcomes } & \multicolumn{4}{|c|}{ Absolute difference per $1000(95 \% \mathrm{Cl})$} & \multirow{2}{*}{$\begin{array}{l}\text { Quality of } \\
\text { evidence } \\
\text { (GRADE) }\end{array}$} \\
\hline & $\begin{array}{l}\text { No of participants } \\
\text { (no of studies) }\end{array}$ & $\begin{array}{l}\text { Risk with no } \\
\text { screening }\end{array}$ & Risk with screening & $\begin{array}{l}\text { Risk ratio } \\
(95 \% \mathrm{Cl})\end{array}$ & \\
\hline Pyelonephritis & $5659(3)$ & 18 & $N S^{*}$ & 0.28 (0.15 to 0.54$)$ & Very low \\
\hline Perinatal mortality & $724(2)$ & 19 & $\mathrm{NS}^{*}$ & 1.21 (0.01 to 102.93$)$ & Very low \\
\hline Spontaneous abortion & $370(1)$ & 55 & $N S^{*}$ & 0.96 (0.41 to 2.27$)$ & Very low \\
\hline Preterm delivery & $722(2)$ & 13 & $N S^{*}$ & 8.70 (0.32 to 240.07$)$ & Very low \\
\hline $\begin{array}{l}\text { Neonatal serious harm: } \\
\text { fetal abnormalities }\end{array}$ & $372(1)$ & 11 & $\mathrm{NS}^{*}$ & $1.50(0.25$ to 8.87$)$ & Very low \\
\hline
\end{tabular}

Frequent screening versus one-time screening

\begin{tabular}{|c|c|c|c|c|c|}
\hline \multirow[b]{2}{*}{ Outcomes } & \multicolumn{4}{|c|}{ Absolute difference per $1000(95 \% \mathrm{Cl})$} & \multirow{2}{*}{$\begin{array}{l}\text { Quality of } \\
\text { evidence } \\
\text { (GRADE) }\end{array}$} \\
\hline & $\begin{array}{l}\text { No of participants } \\
\text { (no of studies) }\end{array}$ & $\begin{array}{l}\text { Risk with one- } \\
\text { time screening }\end{array}$ & $\begin{array}{l}\text { Risk with frequent } \\
\text { screening }\end{array}$ & $\begin{array}{l}\text { Risk ratio } \\
(95 \% \mathrm{Cl})\end{array}$ & \\
\hline Pyelonephritis & $1952(1)$ & 4 & $\mathrm{NS}^{*}$ & 1.09 (0.27 to 4.35$)$ & Very low \\
\hline Perinatal mortality & $1952(1)$ & 49 & $\mathrm{NS}^{*}$ & 1.57 (1.11 to 2.23$)$ & Very low \\
\hline \multicolumn{6}{|c|}{ Treatment versus no treatment/placebo } \\
\hline & & \multicolumn{3}{|c|}{ Absolute difference per $1000(95 \% \mathrm{Cl})$} & \multirow{2}{*}{$\begin{array}{l}\text { Quality of } \\
\text { evidence } \\
\text { (GRADE) }\end{array}$} \\
\hline Outcomes & $\begin{array}{l}\text { No of participants } \\
\text { (no of studies) }\end{array}$ & $\begin{array}{l}\text { Risk with no } \\
\text { treatment }\end{array}$ & Risk with treatment & $\begin{array}{l}\text { Risk ratio } \\
(95 \% \mathrm{Cl})\end{array}$ & \\
\hline Pyelonephritis & $2017(12)$ & 232 & $\begin{array}{l}176 \text { fewer (from } 137 \\
\text { fewer to } 202 \text { fewer) }\end{array}$ & 0.24 (0.13 to 0.41$)$ & Low \\
\hline Perinatal mortality & $1104(6)$ & 40 & $\mathrm{NS}^{*}$ & 0.96 (0.27 to 3.39$)$ & Very low \\
\hline Spontaneous abortion & $379(2)$ & 33 & $\mathrm{NS}^{*}$ & 0.60 (0.11 to 3.10$)$ & Very low \\
\hline Neonatal sepsis & $154(2)$ & 22 & $\mathrm{NS}^{*}$ & 0.22 (0.01 to 4.54$)$ & Very low \\
\hline Preterm delivery & $533(4)$ & 158 & $\mathrm{NS}^{*}$ & 0.22 (0.21 to 1.56$)$ & Very low \\
\hline Low birth weight & $1522(7)$ & 118 & $\begin{array}{l}44 \text { fewer (from } 12 \\
\text { fewer to } 65 \text { fewer) }\end{array}$ & 0.63 (0.45 to 0.90$)$ & Low \\
\hline $\begin{array}{l}\text { Neonatal serious harm: } \\
\text { fetal abnormalities }\end{array}$ & $821(4)$ & 19 & $\mathrm{NS}^{*}$ & 0.49 (0.17 to 1.43$)$ & Very low \\
\hline $\begin{array}{l}\text { Neonatal serious harm: } \\
\text { haemolytic anaemia }\end{array}$ & $265(1)$ & 0 & $\mathrm{NS}^{*}$ & Not estimable & Very low \\
\hline
\end{tabular}

${ }^{*}$ Results failed to show a difference between intervention groups.

GRADE, Grading of Recommendations Assessment, Development and Evaluation; no, number; NS, not significant.

$\mathrm{I}^{2}=31 \%$ versus RR $0.50,95 \%$ CI 0.19 to $1.35 ; \mathrm{I}^{2}=41 \%$ ). The test for subgroup differences was not statistically significant $(\mathrm{p}=0.08)$, but the heterogeneity in each subgroup was reduced and visual inspection of the forest plots

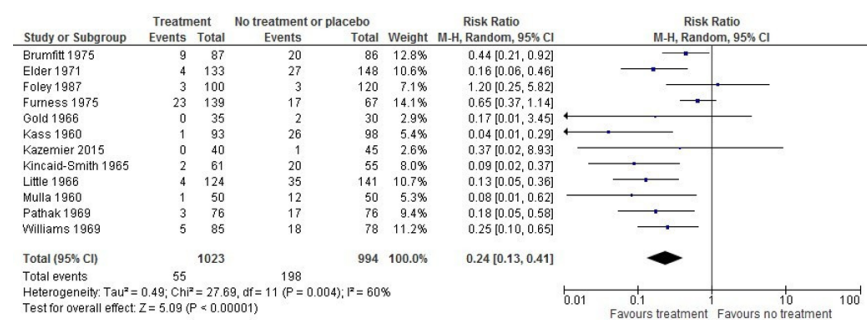

Figure 2 Forest plot of the effect of antibiotic treatment on incidence of pyelonephritis. $\mathrm{M}-\mathrm{H}$, Mantel-Haenszel. suggests a meaningful difference in effect. There was a statistically significant subgroup difference $\left(\chi^{2} p=0.001\right)$ when testing for persistent bacteriuria was done during pregnancy and after delivery (RR $0.11,95 \%$ CI 0.05 to $0.25 ; \mathrm{I}^{2}=0 \%$ ) compared with testing during pregnancy only (RR $0.24,95 \%$ CI 0.13 to $0.41 ; \mathrm{I}^{2}=30 \%$ ) or with testing only after delivery (RR $0.65,95 \%$ CI 0.37 to 1.14 ). Studies that followed women beyond sixweeks after delivery (RR $0.11,95 \% \mathrm{CI} 0.05$ to $0.25 ; \mathrm{I}^{2}=0 \%$ ) found a greater reduction in pyelonephritis than those only following women until delivery or six weeks postdelivery (RR $0.31,95 \%$ CI 0.18 to $\left.0.54 ; \mathrm{I}^{2}=53 \% ; \chi^{2} \mathrm{p}=0.04\right)$.

The funnel plot (online supplementary 7) appeared symmetrical; however, Egger's test was inconclusive $(\mathrm{p}=0.065)$. Twelve studies with small sample sizes limit the 


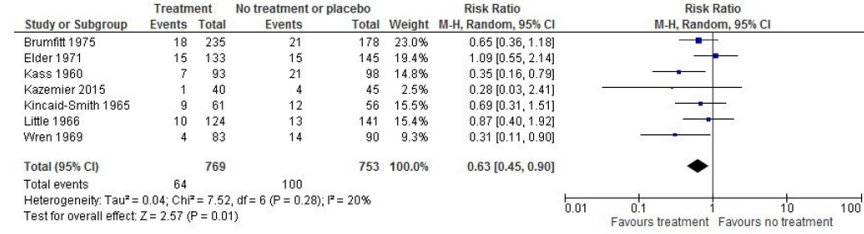

Figure 3 Forest plot of the effect of antibiotic treatment on incidence of babies born at low birth weight. $\mathrm{M}-\mathrm{H}$, MantelHaenszel.

ability to detect or exclude the possibility of small-study bias.

Six studies (1104 women) examined perinatal mortality; one study ${ }^{23}$ was at low ROB, three studies ${ }^{32} 3643$ were at high ROB and two studies ${ }^{37}{ }^{38}$ were unclear. No significant difference was found between groups on perinatal mortality (RR $0.96,95 \%$ CI 0.27 to $3.39 ; \mathrm{I}^{2}=56 \%$; very low quality).

Two studies $^{34} 43$ (379 women) with high ROB reported on spontaneous abortion and found no significant difference between groups (RR $0.60,95 \%$ CI 0.11 to 3.10; $\mathrm{I}^{2}=17 \%$; very low quality).

Two studies ${ }^{23}{ }^{41}$ (154 women) with low ROB reported on neonatal sepsis with no statistically significant difference (very low quality) between groups.

Two studies ${ }^{23}{ }^{41}$ with low ROB and two studies ${ }^{343}$ with high ROB (total 533 women) showed no significant difference between groups on preterm delivery (RR 0.57 , $95 \%$ CI 0.21 to $1.56 ; \mathrm{I}^{2}=70 \%$; very low quality).

Seven studies (1522 women) with two studies ${ }^{23} 38$ at low, three $^{303236}$ at high and one ${ }^{37}$ at unclear ROB examined the effect of treatment on low birth weight (figure 3). There was a statistically significant difference favouring antibiotics (RR 0.63; $95 \%$ CI 0.45 to $0.90 ; \mathrm{I}^{2}=20 \%$; ARR $4.4 \%$; NNT $23,95 \%$ CI 15 to 85 ; low quality).

Four studies (821 women; 2 low ROB, ${ }^{23} 382$ high $\mathrm{ROB}^{32}{ }^{34}$ ) examined the effect of treatment on fetal abnormalities, and found no statistically significant difference between groups (RR $0.49,95 \% \mathrm{CI} 0.17$ to $1.43 ; \mathrm{I}^{2}=0 \%$; very low quality).

One study ${ }^{32}$ (265 women) with high ROB (very low quality) reported no cases of haemolytic anaemia in infants.

No study reported on maternal mortality, maternal sepsis or maternal harms.

\section{CONCLUSIONS AND DISCUSSION}

This paper reports on three SRs to inform recommendations on screening for ASB in pregnancy. Using the GRADE approach, very low quality was found for most outcomes from studies of screening programmes using urine culture, including evidence from one study comparing frequent screening with one-time screening. No direct evidence was found on how women weigh the benefits and harms of screening and/or treatment for ASB and how this valuation might affect their decisions to undergo screening. Low-quality evidence for women with significant bacteriuria provides limited confidence that antibiotic treatment reduces the incidence of pyelonephritis and the number of babies born at low birth weight.

\section{Limitations of evidence base and review}

Many patient and intervention characteristics were inconsistently reported or unreported, making it difficult to infer direct associations between specific risk or intervention factors and outcomes, as well as limiting potential subgroup analyses. While most studies used a urine culture to detect ASB, criteria for defining a positive test were not always clear or reported. One study only included women positive for group B Streptococcus with a lower range criterion for bacteriuria warranting treatment (with many samples considered contaminated species, rather than ASB). Inclusion of these studies may have biased effects of screening programmes and treatment for some outcomes. Outcomes were defined variably among studies. There is potential ROB due to subjectivity of outcomes ascertained by clinical diagnosis (eg, pyelonephritis, when reported among studies, was defined using variable combinations of symptoms).

Much of the evidence came from trials on treatment of bacteriuric women $(2 \%-10 \%$ of screening population), therefore, the results fail to incorporate several effects that would be captured in studies of screening effectiveness (eg, effects on non-screened women who develop symptoms, or on ASB-negative women; effects from non-adherence to screening protocol). Only three studies explicitly reported patients as exclusively asymptomatic pregnant women; among treated patients, the beneficial effects may be larger among symptomatic women compared with asymptomatic women. Early stopping due to low incidence of primary outcomes in one study $^{23}$ may have biased effects of treatment. The finding of a greater reduction in pyelonephritis among women who were followed up beyond post partum suggests that a longer follow-up period within a screening programme may prevent later development of bacteriuria (and subsequent pyelonephritis) when there is concern regarding adverse maternal effects.

The majority of studies were published in the $1960 \mathrm{~s}$ to 1980 s, predating current obstetric practices having, for example, better recognition of risk factors for UTIs and other pregnancy complications, prompt treatment of symptoms, a broader range of antibiotic options and improved ascertainment of maternal and neonatal outcomes.

\section{Comparison with other reviews}

Published after the completion of this review, an SR by Angelescu et $a t^{44}$ that examined benefits and harms of screening for ASB in pregnancy found no trials on screening effectiveness. The review authors included four RCTs focused on treatment of ASB. ${ }^{23} 313942$ These authors limited inclusion to studies reporting exclusively on treatment in asymptomatic women. We included studies that likely included some women with symptoms, and found 
no meaningful difference for this variable in subgroup analysis. Angelescu et $a t^{44}$ examined some intervention characteristics (eg, treatment regimen and adjunct treatments) and outcomes (eg, lower UTI, very low birth weight $<1500 \mathrm{~g}$ ) that were not included in our review. They concluded that there was no reliable evidence on the benefits and harms of screening to support routine screening for ASB using urine culture in pregnant women. ${ }^{44}$

\section{Future research}

High-quality RCTs of the effectiveness of screening programmes should be undertaken. Current evidence provides uncertainty regarding: (1) applicability to current practice, (2) adequate collection and reporting of harms and (3) modern and clearly defined outcomes. Because routine screening practices suggest limited clinical equipoise, contemporary studies using a pragmatic preference-based/tolerant screening trial design (eg, those without a preference towards/against screening are randomised while others self-select one arm) should be adopted.

Better information is needed to determine whether there are important moderating factors for ASB screening, as we attempted to examine in comparing different screening methods/algorithms. Subgroup analyses of studies using one urine culture versus at least one additional confirmatory culture had some credibility but were limited to reliance on between-study effects. Studies directly examining this, and other factors such as different thresholds for treatment, could provide high-quality data and be informative for how to maximise benefit. Enhanced culture protocols (eg, expanded spectrum) for detecting the most clinically relevant uropathogens are emerging, ${ }^{45} 46$ and if found to consistently provide better detection of these micro-organisms than standard urine culture, studies comparing screening programmes differing by these methods are encouraged to determine if they also predict how well treatment reduces the risk for pyelonephritis and other pregnancy complications in asymptomatic women.

More evidence or information about how women weigh the benefits and harms of screening and treatment for ASB in pregnancy would be valuable. It may be useful to use deliberative processes or focus groups to facilitate patients' understanding of results on such benefits and harms; this may be informative to determine whether it is critical to better engage patients in shared decision-making.

Acknowledgements Canadian Task Force on Preventive Health Care members who contributed to the protocol development and discussion of results included the Asymptomatic Bacteriuria Guideline Working Group: Ainsley Moore, Roland Grad, Stéphane Groulx, Kevin Pottie, Brett Thombs and Marcello Tonelli. Public Health Agency of Canada Global Health and Guidance Division staff involved with the review and the guideline included Marion Doull, Alejandra Jaramillo Garcia, Susan Courage and Nicki Sims-Jones. Knowledge Translation Program, Li Ka Shing Knowledge Institute, St. Michael's Hospital, for their contributions to patient engagement and knowledge translation work related to the protocol development, including the participants of the patient engagement activities. We are grateful to the following reviewers for their feedback on this evidence review, as well as those individuals who preferred to remain anonymous: Anne Biringer, Linda Brubaker, Venu Jain, Jennifer Marandola, Deborah Money, Lindsay Nicolle and Fiona Smaill. We would like to acknowledge Tara Landry for peer reviewing the search strategy for screening effectiveness, Lynn Hampson for conducting search updates for treatment trials, Allison Gates for assistance with quality assessment and data extraction and verification for the French study, Hamza Jafri for assistance with screening, Megan Nuspl for assistance with screening grey literature, Tara MacGregor for assistance with DistillerSR and Evans Kwak and MacKinna Hauff for assistance with article retrieval. We would like to thank Konstanze Angelescu, Brenda Kazemier, Mary Ann Rhode and Yeşim Uncu for their assistance in providing study details.

Contributors LH, AW, JP, MS, MG, RF, KS and BV critically reviewed and contributed to drafts of the report. RF conducted the literature searches. AW, JP, MS, MG, KS and BV conducted screening, quality assessments, data extraction and analysis. AW, JP, LH, MS, KS and BV contributed to interpretation of results. All of the authors approved the final version of this report.

Funding Funding for the Evidence Review is provided by the Public Health Agency of Canada.

Disclaimer The views of the funding bodies have not influenced the content of the guideline; competing interests have been recorded and addressed. The views expressed in this article are those of the authors and do not necessarily represent those of the Public Health Agency of Canada.

Competing interests All of the authors report grants from the Public Health Agency of Canada during the conduct of the study.

Patient consent for publication Not required.

Provenance and peer review Not commissioned; externally peer reviewed.

Data sharing statement All authors had full access to all of the data in the study and can take responsibility for the integrity of the data and the accuracy of the interpretation. Data for this systematic review are available from the corresponding author on reasonable request.

Open access This is an open access article distributed in accordance with the Creative Commons Attribution Non Commercial (CC BY-NC 4.0) license, which permits others to distribute, remix, adapt, build upon this work non-commercially, and license their derivative works on different terms, provided the original work is properly cited, appropriate credit is given, any changes made indicated, and the use is non-commercial. See: http://creativecommons.org/licenses/by-nc/4.0/.

\section{REFERENCES}

1. Glaser AP, Schaeffer AJ. Urinary tract infection and bacteriuria in pregnancy. Urol Clin North Am 2015;42:547-60.

2. Schnarr J, Smaill F. Asymptomatic bacteriuria and symptomatic urinary tract infections in pregnancy. Eur J Clin Invest 2008;38(Suppl 2):50-7.

3. Ipe DS, Sundac L, Benjamin WH, et al. Asymptomatic bacteriuria: prevalence rates of causal microorganisms, etiology of infection in different patient populations, and recent advances in molecular detection. FEMS Microbiol Lett 2013;346:1-10.

4. Smaill FM, Vazquez JC. Antibiotics for asymptomatic bacteriuria in pregnancy. Cochrane Database Syst Rev 2015:CD000490.

5. Guinto VT, De Guia B, Festin MR, et al. Different antibiotic regimens for treating asymptomatic bacteriuria in pregnancy. Cochrane Database Syst Rev 2010;9:CD007855.

6. Patterson TF, Andriole VT. Bacteriuria in pregnancy. Infect Dis Clin North Am 1987;1:807-22.

7. Canadian Agency for Drugs and Technologies in Health. Grey matters: a practical tool for searching health-related grey literature. Ottawa, ON, 2015

8. Evidence Partners Inc. Distiller (DistillerSR systematic review software program). http://systematic-review.net/ (cited: 20 Dec 2017).

9. Centre for Evidence-based Management (CEBMa). Critical appraisal of a cross-sectional study (survey). https://www.cebma.org/ resources-and-tools/what-is-critical-appraisal/ (cited: 28 Jan 2017).

10. Higgins JP, Green S. Section 8. Assessing risk of bias in included studies. from:http://handbook.cochrane.org (cited: 20 Dec 2017).

11. Guyatt GH, Oxman AD, Santesso N, et al. GRADE guidelines: 12. Preparing summary of findings tables-binary outcomes. J Clin Epidemiol 2013;66:158-72. 
12. Egger M, Davey Smith G, Schneider M, et al. Bias in meta-analysis detected by a simple, graphical test. BMJ 1997;315:629-34.

13. Guyatt GH, Oxman AD, Schünemann HJ, et al. GRADE guidelines: a new series of articles in the Journal of Clinical Epidemiology. J Clin Epidemiol 2011;64:380-2.

14. Hsu J, Brożek JL, Terracciano L, et al. Application of GRADE: making evidence-based recommendations about diagnostic tests in clinical practice guidelines. Implement Sci 2011;6:62.

15. Guyatt GH, Oxman AD, Kunz R, et al. GRADE guidelines 6 . Rating the quality of evidence-imprecision. $J$ Clin Epidemiol 2011;64:1283-93.

16. McMaster University. GRADEpro (GRADEpro GDT computer software). https://gradepro.org (cited: 20 Dec 2017).

17. Guyatt G, Oxman AD, Akl EA, et al. GRADE guidelines: 1 . Introduction-GRADE evidence profiles and summary of findings tables. J Clin Epidemiol 2011;64:383-94.

18. Gérard J, Blazquez G, Mounac M. [Importance of systematic research of urinary infection in pregnant women and the cost of its detection. Proposal for a practical approach]. J Gynecol Obstet Biol Reprod 1983;12:243-51.

19. Gratacós E, Torres PJ, Vila J, et al. Screening and treatment of asymptomatic bacteriuria in pregnancy prevent pyelonephritis. $J$ Infect Dis 1994:169:1390-2.

20. Rhode MA, Shapiro H, Jones OW. Indicated vs. routine prenatal urine chemical reagent strip testing. J Reprod Med 2007;52:214-9.

21. Uncu Y, Uncu G, Esmer A, et al. Should asymptomatic bacteriuria be screened in pregnancy? Clin Exp Obstet Gynecol 2002;29:281-5.

22. Butters $L$, Howie CA. Awareness among pregnant women of the effect on the fetus of commonly used drugs. Midwifery 1990;6:146-54.

23. Kazemier BM, Koningstein FN, Schneeberger C, et al. Maternal and neonatal consequences of treated and untreated asymptomatic bacteriuria in pregnancy: a prospective cohort study with an embedded randomised controlled trial. Lancet Infect Dis 2015;15:1324-33.

24. Sharma R, Kapoor B, Verma U. Drug utilization pattern during pregnancy in North India. Indian J Med Sci 2006;60:277-87.

25. Lupattelli A, Picinardi M, Einarson A, et al. Health literacy and its association with perception of teratogenic risks and health behavior during pregnancy. Patient Educ Couns 2014;96:171-8.

26. Mashayekhi SO, Dilmaghanizadeh M, Fardiazar Z, et al. Study of awareness among pregnant women of the effects of drugs on the fetus and mother in Iran. Health Policy 2009;91:89-93.

27. Nordeng H, Ystrøm E, Einarson A. Perception of risk regarding the use of medications and other exposures during pregnancy. Eur J Clin Pharmacol 2010;66:207-14.

28. Sanz E, Gómez-López T, Martínez-Quintas MJ. Perception of teratogenic risk of common medicines. Eur J Obstet Gynecol Reprod Biol 2001;95:127-31.
29. Twigg MJ, Lupattelli A, Nordeng $\mathrm{H}$. Women's beliefs about medication use during their pregnancy: a UK perspective. Int J Clin Pharm 2016;38:968-76.

30. Brumfitt $W$. The effects of bacteriuria in pregnancy on maternal and fetal health. Kidney Int Supp/ 1975;4(Supp 4):113.

31. Elder HA, Santamarina BA, Smith SA, et al. Use of sulfasymazine in the treatment of bacteriuria of pregnancy. Antimicrob Agents Chemother 1966;6:142-8.

32. Elder HA, Santamarina BA, Smith S, et al. The natural history of asymptomatic bacteriuria during pregnancy: the effect of tetracycline on the clinical course and the outcome of pregnancy. Am J Obstet Gynecol 1971;111:441-62.

33. Foley ME, Farquharson R, Stronge JM. Is screening for bacteriuria in pregnancy worthwhile? Br Med J 1987;295:270.

34. Furness ET, McDonald PJ, Beasley NV. Urinary antiseptics in asymptomatic bacteriuria of pregnancy. $N Z$ Med $J$ 1975;81:417-9.

35. Gold EM, Traub FB, Daichman I, et al. Asymptomatic bacteriuria during pregnancy. Obstet Gynecol 1966;27:206-9.

36. Kass $\mathrm{E}$. The role of asymptomatic bacteriuria in the pathogenesis of pyelonephritis. In: Quinn EL, Kass EH, eds. Biology of pyelonephritis. Boston: Little Brown and Company, 1960:399-412.

37. Kincaid-Smith P, Bullen M. Bacteriuria in pregnancy. Lancet 1965;1:395-9.

38. Little PJ. The incidence of urinary infection in 5000 pregnant women. Lancet 1966;2:925-8.

39. Mulla N. Bacteriuria in pregnancy. Obstet Gynecol 1960;16:89-92.

40. Pathak UN, Tang K, Williams LL, et al. Bacteriuria of pregnancy: results of treatment. $J$ Infect Dis 1969;120:91-103.

41. Thomsen AC, Mørup L, Hansen KB. Antibiotic elimination of group-B streptococci in urine in prevention of preterm labour. Lancet 1987;1:591-3.

42. Williams GL, Campbell H, Davies KJ. Urinary concentrating ability in women with asymptomatic bacteriuria in pregnancy. Br Med $\mathrm{J}$ 1969;3:212-5.

43. Wren BG. Subclinical renal infection and prematurity. Med J Aust 1969;2:596-600.

44. Angelescu K, Nussbaumer-Streit B, Sieben W, et al. Benefits and harms of screening for and treatment of asymptomatic bacteriuria in pregnancy: a systematic review. BMC Pregnancy Childbirth 2016;16:336.

45. Hilt EE, McKinley K, Pearce MM, et al. Urine is not sterile: use of enhanced urine culture techniques to detect resident bacterial flora in the adult female bladder. $J$ Clin Microbiol 2014;52:871-6.

46. Price TK, Dune T, Hilt EE, et al. The Clinical Urine Culture: Enhanced Techniques Improve Detection of Clinically Relevant Microorganisms. J Clin Microbiol 2016;54:1216-22. 